\title{
Influence of calcined mud on the mechanical properties and shrinkage of self-compacting concrete
}

\section{Influence de la vase calcinée sur les propriétés mécaniques et le retrait des bétons autoplaçants}

Taieb Fatima ${ }^{1}$, Belas Nadia, Belaribi Omar, Belguesmia Khalil and Hadj Sadok Rachid

Laboratoire Construction Transport et Protection de l'Environnement, Département de Génie Civil et d'architecture, Université de Mostaganem, Mostaganem, Algérie

nadakhalifa27@gmail.com

\begin{abstract}
The use of SCC has a particular interest in terms of sustainable development. Indeed, their specific formulation leads to a greater volume of dough than for common concretes, thus, a larger quantity of cement. However, for economical, ecological and technical reasons, it is sought to limit their cement content [1]. It is therefore necessary to almost always use mineral additions as a partial replacement for cement because the technology of self-compacting concretes can consume large quantities of fines, in this case calcinated mud issued from dams dredging sediments that can give and/or ameliorate characteristics and performances of this type of concretes. Four SCCs had been formulated from the same composition where the only percentage of calcinated mud of Chorfa (west of Algeria) dam changed (0\%, 10\%, 20\% and 30\%). The effect of calcinated mud on characteristics at fresh state of SCC according to AFGC was quantified. Mechanical strengths and shrinkage deformation (total, autogenous, drying) were evaluated. The results show the possibility to make SCCs with different dosages of calcinated mud having strengths that can defy those of the control SCC. The analysis of free deformations indicates the beneficial impact of the mud by contributing to decrease the amplitudes of the shrinkage compared to those of the control SCC.
\end{abstract}

Résumé. L'utilisation des BAP revêt un intérêt particulier en termes de développement durable. En effet, leur formulation spécifique conduit à un volume de pâte plus important que pour des bétons traditionnels et donc, a priori, à une plus grande quantité de ciment. Or, pour des raisons économiques et des considérations d'ordre écologiques et techniques, on cherche à limiter leur teneur en ciment [1]. II est donc nécessaire d'utiliser quasi systématiquement des additions minérales en remplacement partiel au ciment car la technologie des bétons autoplaçants est capable de consommer d'énormes quantités de fines en l'occurrence la vase calcinée issue des sédiments de dragage des barrages, qui à son tour pourrait apporter et/ou améliorer des caractéristiques et performances de ce type de bétons. Quatre BAP ont été formulés à partir d'une même composition où seul le pourcentage de la vase calcinée issue des sédiments de dragage du barrage de Chorfa (ouest algérien) change (0\%, 10\%, 20\% et 30\%). L'effet de la vase sur les caractéristiques des BAP à l'état frais a été quantifié. Des résistances mécaniques et les déformations de retrait (total, endogène et de dessiccation) ont été évaluées. Les résultats montrent la possibilité de confectionner des BAP avec différents dosages en vase calcinée ayant des résistances qui peuvent défier celles du BAP témoin. L'analyse des déformations libres indique l'impact bénéfique de la vase en contribuant à diminuer les amplitudes du retrait par rapport à celles du BAP témoin. 


\section{Introduction}

L'intérêt des bétons autoplaçants et leur rapide succès auprès des maîtres d'œuvre tient à leur facilité de mise en œuvre qui permet d'améliorer la qualité des travaux en termes de finition notamment pour les ouvrages de formes complexes ou fortement ferraillés [2]. Cependant, le contrôle du retrait de ce type de béton reste un problème majeur non résolu compromettant l'élargissement de son domaine d'utilisation.

L'objectif principal de cette recherche est d'étudier les propriétés des bétons autoplaçants à base de différents pourcentages de substitution en sédiments de dragage traités en l'occurrence, la vase du barrage Chorfa, et ce à l'état durci à savoir les résistances mécaniques en compression et les variations dimensionnelles dues au retrait, et ce en comparant les résultats des essais à ceux des éprouvettes de BAP de référence.

\section{Matériaux et Formulations}

\subsection{Matériaux}

\subsubsection{Ciment}

Un ciment $42.5 \mathrm{~N}$ ES a été utilisé pour les différentes formulations, sa surface Blaine est de $3200 \mathrm{~cm}^{2} / \mathrm{g}$ et sa masse volumique absolue est de $3.17 \mathrm{~g} / \mathrm{cm}^{3}$. La composition minéralogique du clinker est présentée dans le tableau 1.

Tableau 1. Composition minéralogique du clinker selon Bogue.

\begin{tabular}{|c|c|c|c|c|}
\hline $\begin{array}{c}\text { Les } \\
\text { minéraux }\end{array}$ & $\mathrm{C}_{3} \mathrm{~S}$ & $\mathrm{C}_{2} \mathrm{~S}$ & $\mathrm{C}_{3} \mathrm{~A}$ & $\mathrm{C}_{4} \mathrm{AF}$ \\
\hline$\%$ & 60.63 & 17.29 & 1.82 & 15.66 \\
\hline
\end{tabular}

\subsubsection{Vase}

La vase utilisée dans la formulation du béton autoplaçant a été prélevée du barrage de Chorfa II situé au niveau de la wilaya de Mascara dans l'ouest Algérien. Elle a subi un traitement thermique par calcination dans un four lent à température de $750+5 \mathrm{C}^{\circ}$ réglé à $5 \mathrm{C}^{\circ} /$ minute pendant 5 heures après qu'elle soit étuvée, broyée et tamisée à 80 $\mu \mathrm{m}$. La surface spécifique et la densité de la vase calcinée sont respectivement $8310 \mathrm{~cm}^{2} / \mathrm{g}$ et $2.74 \mathrm{~g} / \mathrm{cm}^{3}$. Ses caractéristiques chimiques sont données dans le tableau 2.

Tableau 2. Composition chimique de la vase calcinée

\begin{tabular}{|c|c|}
\hline Oxydes & Teneur (\%) \\
\hline $\mathrm{SiO}_{2}$ & 48.39 \\
\hline $\mathrm{CaO}$ & 17.03 \\
\hline $\mathrm{Fe}_{2} \mathrm{O}_{3}$ & 6.82 \\
\hline $\mathrm{Al}_{2} \mathrm{O}_{3}$ & 15.63 \\
\hline $\mathrm{MgO}$ & 2.85 \\
\hline $\mathrm{Cl}$ & 0.066 \\
\hline $\mathrm{K}_{2} \mathrm{O}$ & 2.3 \\
\hline $\mathrm{SO}_{3}$ & 0.23 \\
\hline
\end{tabular}

\subsubsection{Granulats}

Les granulats utilisés sont des granulats concassés calcaire provenant de la carrière de Hasnaoui à Sidi Belabbas (Ouest Algérien) de classes $0 / 3$ et $3 / 8$ et 8/15. Leur densité est respectivement $2.65,2.64$ et 2.67 . Le sable de mer $0 / 2$ de nature siliceuse provenant de la carrière de Sidi-Lakhdar à Mostaganem (Ouest Algérien) avec une densité de 2.55 .

\subsubsection{Adjuvant}

Un seul type d'adjuvant a été utilisé à savoir le Sika Viscocrète Tempo 12, un super plastifiant haut réducteur d'eau polyvalent de nouvelle génération à base de copolymère acrylique avec une densité de $1.06 \pm 0.01$ et de 28.0 à $31.0 \%$ d'extrait sec.

\subsection{Formulations des BAP}

Dans l'élaboration des formulations des BAP le ciment a été remplacé partiellement par 10, 20 et $30 \%$ de vase calcinée. Au total, quatre BAP ont été confectionnés avec un rapport $\mathrm{E} / \mathrm{L}$ constant égal à 0.5 , leurs compositions sont données dans le tableau 3. La caractérisation à l'état frais des bétons autoplaçants s'est limité aux essais recommandés par l'AFGC (AFGC ,2008) : étalement au cône (SF), écoulement à la boite en L et stabilité au tamis (Sr). Les résultats à l'état frais donnés dans le tableau 3 montrent la possibilité de formuler des BAP avec la vase calcinée qui ont été qualifiés d'autoplaçants selon les spécificités de l'AFGC [3].

Tableau 3. Composition et propriétés à l'état frais des BAP

\begin{tabular}{|c|c|c|c|c|}
\hline $\begin{array}{c}\text { Designation } \\
\text { BAP }\left(\mathrm{kg} / \mathrm{m}^{3}\right)\end{array}$ & BAPT & BAP10VC & BAP20VC & BAP30VC \\
\hline E/L & 0.5 & 0.5 & 0.5 & 0.5 \\
\hline Ciment & 451 & 405.9 & 360.8 & 315.7 \\
\hline Vase & $/$ & 45.1 & 90.2 & 135.3 \\
\hline Eau & 225.5 & 225.5 & 225.5 & 225.5 \\
\hline Adjuvant & 2.3 & 3.56 & 4.56 & 5.70 \\
\hline Sm (0/2) & 558 & 558 & 558 & 558 \\
\hline Sc (0/3) & 248 & 248 & 248 & 248 \\
\hline G (3/8) & 330 & 330 & 330 & 330 \\
\hline G (8/15) & 501 & 501 & 501 & 501 \\
\hline
\end{tabular}




\begin{tabular}{|c|c|c|c|c|}
\hline $\mathrm{SF}(\mathrm{cm})$ & 69.75 & 72.75 & 68.25 & 73.5 \\
\hline $\begin{array}{c}\mathrm{L}-\mathrm{Box} \\
\left(\mathrm{H}_{2} / \mathrm{H}_{1}\right)\end{array}$ & 87.25 & 83.33 & 81.5 & 89.8 \\
\hline $\mathrm{Sr}(\%)$ & 6.86 & 6.64 & 5.14 & 1 \\
\hline
\end{tabular}

\section{Essais expérimentaux}

\subsection{Essai de compression simple}

Les tests ont été réalisés sur des éprouvettes cubiques 100 mm x 100 mm conservées dans l'eau dès leur démoulage jusqu'aux échéances d'écrasement.

\subsection{Retrait libre}

Nous savons que l'élément le plus influant sur le retrait c'est la quantité d'eau utilisée. Pour cela et pour mieux cerner le problème, nous avons gardé le rapport $\mathrm{E} / \mathrm{L}=0,5$ fixe et la quantité d'eau $225.5 \mathrm{~kg} / \mathrm{m}^{3}$ fixe.

Les mesures du retrait endogène et du retrait total ont été réalisées $24 \mathrm{~h}$ après le début du malaxage pour chaque composition, les variations dimensionnelles ont été effectués avec un comparateur sur trois éprouvettes conservées dans une salle maintenue à $20^{\circ} \mathrm{C}$ et $50 \%$ d'humidité relative (retrait total) et sur trois autres éprouvettes protégées par un film adhésif pour empêcher tout échange hydrique (retrait endogène). La différence de ces deux retraits permet d'obtenir le retrait de dessiccation.

\section{Résultats et Discussion}

\subsection{Résistances mécaniques}

L'évolution de la résistance en compression en fonction du temps (cure dans l'eau pendant 3, 7, 14, 28, 60 et 90 jours) et du pourcentage de la vase calcinée est donnée par la figure 1. Les résultats montrent que pour tous les BAP étudiés, la résistance en compression augmente avec l'âge en fonction du dosage de vase calcinée.

Les BAP à base de vase affichent de bonnes performances mécaniques par rapport au BAP témoin.

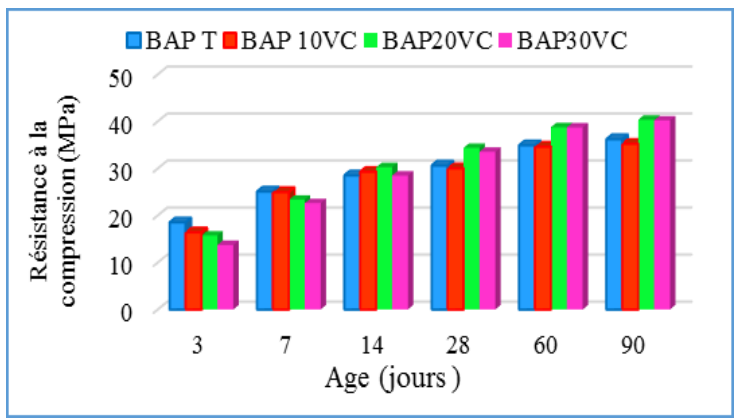

Fig. 1. Evolution de la résistance à la compression des BAP
La figure 1 montre que jusqu'à l'âge de 14 jours les résistances développées pour tous les BAP semblent très proches néanmoins au-delà de cette échéance deux cinétiques se distinguent avec une supériorité pour les BAP à $20 \%$ et $30 \%$ d'ajout.

A 28, 60 et 90 jours, on remarque déjà une différence de $4 \mathrm{MPa}$ entre les deux BAP $(20 \mathrm{VC}, 30 \mathrm{VC})$ et le BAP témoin, cette différence n'est que la conséquence de l'activité pouzzolanique.

A partir de la figure 2 qui montre les rapports entre les résistances des $\mathrm{BAPVC}$ et $\mathrm{BAP} \mathrm{T}$, il est à constater que les deux BAP (20VC et 30VC) affichent les meilleures performances par rapport au BAP témoin

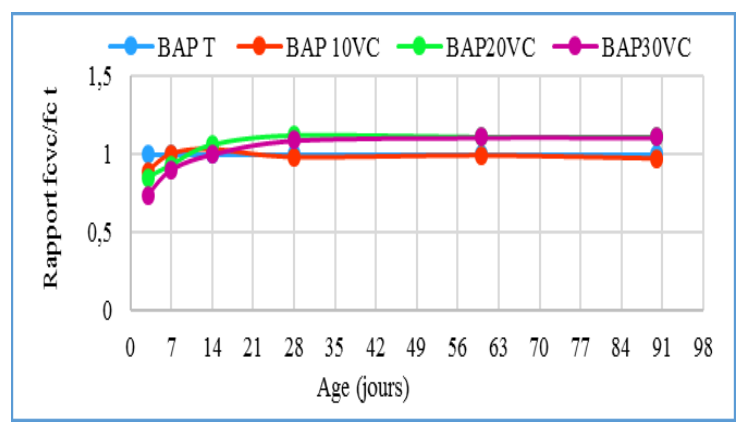

Fig. 2. Variation des résistances en compression des BAPVC/BAPT

En général, la résistance à la compression du BAP à base de vase calcinée est supérieur à celle du BAP témoin pour un même rapport $\mathrm{E} / \mathrm{L}$ et même quantité d'eau.

\subsection{Déformations différées}

\subsubsection{Retrait endogène}

Les évolutions du retrait endogène des BAP mesuré sur des éprouvettes $7 \times 7 \times 7 \times 28 \mathrm{~cm}^{3}$ sont présentées sur la figure 3 .

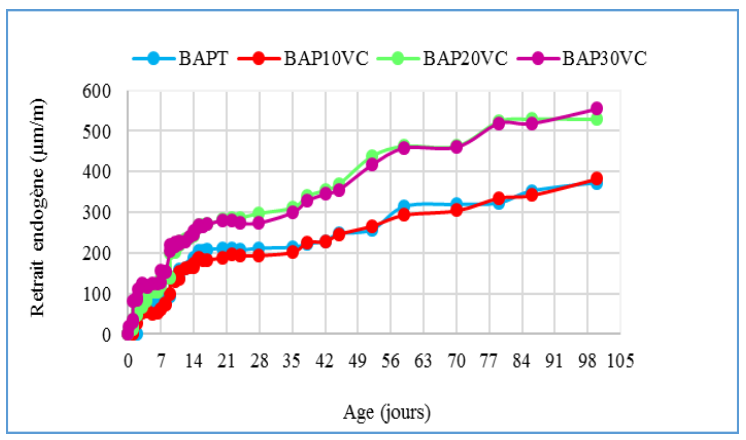

Fig.3. Evolution du retrait endogène en fonction du temps

Il apparaît dans la figure 3, que les cinétiques des déformations du retrait endogène des bétons BAPVC, sont assez semblables, elles présentent des allures similaires et presque identiques aux premiers âges et 
commencent à se différencier au fur à mesure que nous avançons dans le temps [4]. Les BAP à base de $20 \%$ et $30 \%$ de vase présentent les déformations les plus élevées, dès les premiers jours et pour toute la durée des essais. $\mathrm{Au}$ contraire les BAP (témoin et à $10 \%$ de vase) présentent les plus faibles retraits endogènes. Si l'on se base sur la définition du retrait endogène qui résulte du fait que le volume des hydrates formés est légèrement inférieur au volume des composants initiaux (eau + ciment), ce processus entraîne une diminution de volume après la prise du béton, alors on peut clairement dire que les bétons contenant la vase calcinée nous révèlent la formation d'autres types d'hydrates autres que ceux dus à l'hydratation du ciment, sans négliger l'effet physique de cette même vase suite à sa grande finesse [5]. Les additions minérales et les adjuvants contenus dans la pâte des BAP ont surtout un effet sur la cinétique de la contraction Le Chatelier et peu sur son amplitude finale [6], ce qui est le cas pour nos mélanges à base de $20 \%$ et de $30 \%$ de vase calcinée.

Il est admis que le retrait endogène est une conséquence des changements d'HR dans les pores [7]. En fonction de la structure des pores et de l'eau disponible; différents mécanismes sont déclenchés [7].

Le BAP10VC présente le plus faible retrait endogène ; ce qui est remarqué avec les faibles pertes de masse endogène obtenues pour ce mélange. Le retrait endogène présente un même développement en fonction des résistances mécaniques; il est proportionnel à la résistance en compression du béton [1] ; et ce que nous avons déjà vu dans les résultats de compression.

\subsubsection{Retrait de dessiccation}

On constate sur la figure 4, que le BAP 10VC présente les valeurs les plus élevées après la première semaine de durcissement. En revanche les BAP à base de $20 \%$ et $30 \%$ de vase présentent des valeurs semblables et inférieures par rapport à celles du témoin, à 28 jours on constate que le retrait des BAP20VC et 30VC sont inférieurs de 59 à $31 \%$ respectivement par rapport au BAPT, cette phase illustrera le début d'une certaine activité pouzzolanique.

Cela peut être expliqué par le rôle à la fois physique de la vase si l'on considère les résultats des autres chercheurs qui affirment que la présence des ajouts minéraux à des taux convenables fait diminuer le retrait de dessiccation grâce à leur contribution à l'amélioration de la microstructure [5]. On peut dire que l'addition de la vase calcinée réduit le retrait de dessiccation et par conséquent ce matériaux apporte un plus aux problèmes de retrait des BAP [8].

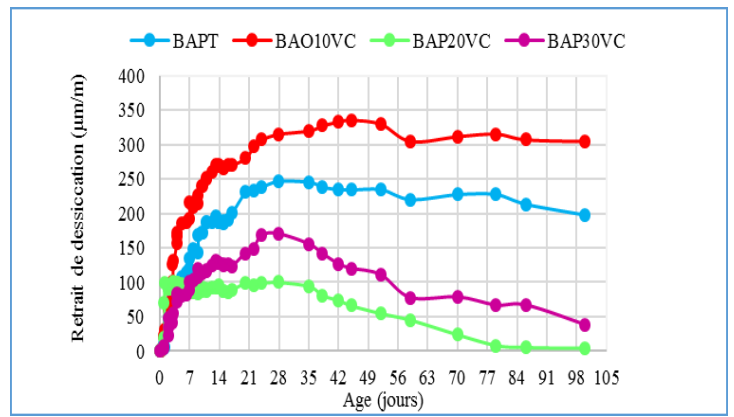

Fig.4. Évolution du retrait de dessiccation en fonction du temps

\subsubsection{Retrait Total}

Les courbes de la figure 5 montrent l'évolution du retrait total dans le temps des éprouvettes de BAP non confinées.

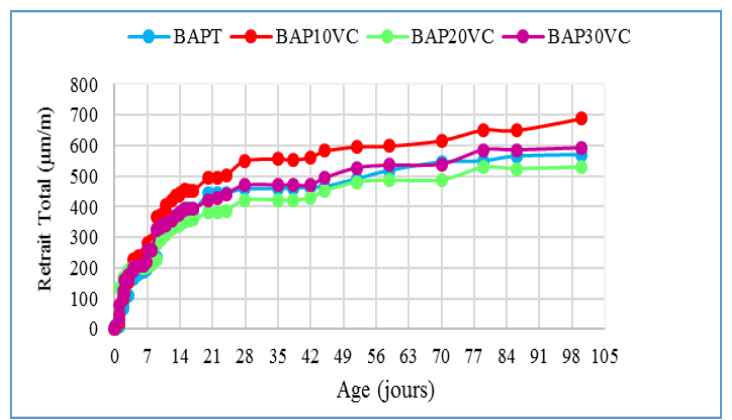

Fig.5. Évolution du retrait total en fonction du temps

Le retrait total évolue très rapidement pour tous les types d'éprouvettes conservées à l'air libre, à cause de la taille de ces dernières qui rend la dessiccation plus favorable [4]. Les évolutions présentent des allures similaires et presque identiques aux premiers âges et commencent à se différencier dans le temps. Le BAP20VC présente le plus faible retrait total. La figure 5 montre une évolution de déformation similaire pour le BAPT et le BAP à base de $30 \%$ de vase calcinée, car au très jeune âge nous avons du mal à distinguer entre les graphes représentatifs de chaque béton. Le BAP10VC présente les déformations totales les plus élevées, dès les premiers jours et pour toute la durée des essais.

Si l'on considère le fait que les mesures se font à l'échelle du micromètre alors il est plus facile de dire qu'à long terme la composition des BAP étudiés n'a pas une grande influence sur le retrait total [4].

\subsubsection{Perte de masse}

Afin de mieux comprendre les interprétations précédentes du retrait nous avons mesuré en parallèle la perte de masse exprimée en \%.

Les pertes de masse ont été mesurées sur les éprouvettes destinées aux mesures du retrait total et endogène. Les résultats sont représentés par les figures 6 et 7 .

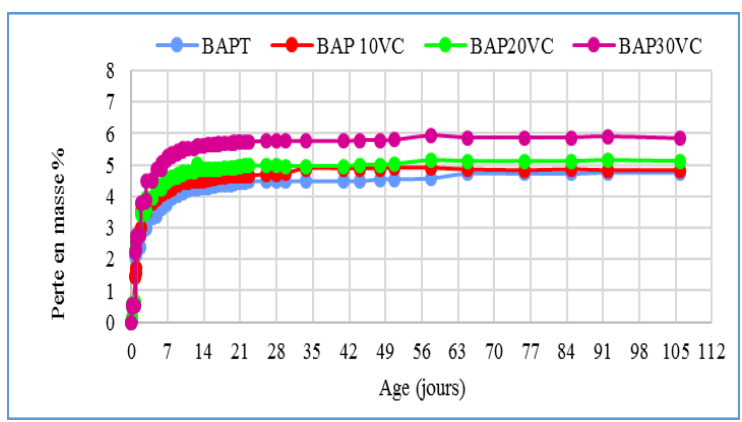


Fig.6. Évolution de la perte de masse totale en fonction du temps

D'après la figure 6 , on remarque que le BAPT et les deux BAP de $10 \%$ et $20 \%$ de vase calcinée exposés au retrait total ont la même cinétique de perte de masse sauf le BAP à base de $30 \%$ de vase se détache par rapport aux autres en développant plus de perte. L'analyse des courbes montre que la plus grande partie du retrait a lieu avant 28 jours environ, au-delà de cet âge, il est stabilisé. Ceci s'explique par le fait que la réaction d'hydratation est une réaction exothermique qui participe d'une part au départ d'eau par évaporation d'eau vers l'ambiance extérieure et d'autre part par consommation dans l'action d'hydratation du ciment. Cette échéance, 90 jours, correspond assez bien au ralentissement du séchage, observé sur la perte de masse. Au-delà, le retrait de séchage n'évolue quasiment plus [9].

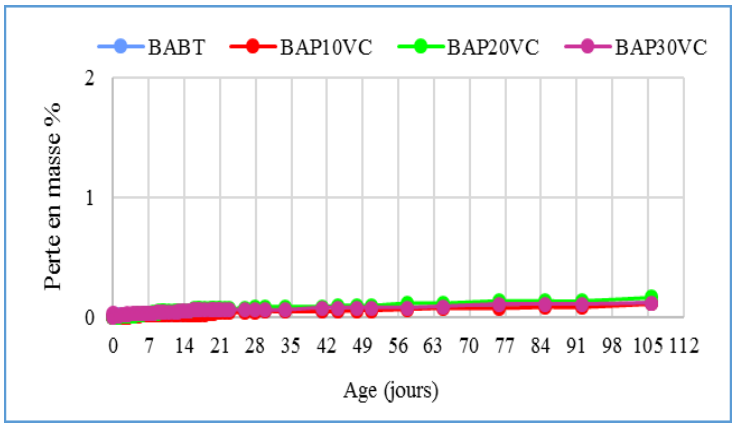

La figure 7 montre le développement en perte de masse de BAP qui est mesuré sur des éprouvettes exposées à un retrait endogène.

Fig.7. Évolution de la perte de masse endogène en fonction du temps

Ces pertes de masse sont causées par la consommation interne d'eau par l'hydratation du ciment et la vase. On observe que tous les BAP évoluent de la même façon en fonction du temps. Les BAP (témoin, $10 \%$ et $30 \%$ de vase calcinée) croît dans un fuseau très serré jusqu'à l'âge de 60 jours où les valeurs commencent à converger, excepté pour le BAP à $20 \%$ qui présente une évolution à part.

Le dosage de $10 \%$ semble optimal pour des pertes en masse minimales et par conséquent un retrait réduit et ce que nous avons déjà vu dans la courbe du retrait endogène.

\section{Conclusion}

Cette étude avait pour objectif de comparer les propriétés mécaniques de bétons autoplaçants à base de différents pourcentages de vase calcinée avec celles du béton témoin utilisé comme référence.
En ce qui concerne les résistances mécaniques, celles-ci augmentent avec l'augmentation du pourcentage de la vase calcinée dans le béton, à long terme.

La présence de la vase à des taux de $20 \%$ et $30 \%$ fait diminuer le retrait de dessiccation et total, cela est le résultat de leur contribution à l'amélioration de la microstructure.

On peut en conclure qu'une substitution de $30 \%$ du ciment par la vase est le taux le plus intéressant qui s'avère optimal, puisque c'est le taux qui satisfait les deux critères d'amélioration de résistance et réduction du retrait

\section{References}

1. Schwartzentruber L et Torrenti J., Le grand livre des bétons, Paris, Editions Le moniteur, (2014)

2. Bernardini C., Les bétons autoplaçants, Paris., Presses Ponts et Chaussées, Août (2011).

3. AFGC Bétons Autoplaçants Recommandations provisoires, Association française de Génie Civil, (2008)

4. Bouhamou.N, Etude du comportement d'un béton autoplaçant à base de vase de dragage vis-à-vis du retrait, Colloque 01 Ecomatériaux, Matériaux 2014, 24-28 Novembre (2014).

5. Chalarem. N., Caractérisation des BAP à base de sédiments de dragage vis-à-vis du retrait au jeune âge, Mémoire de fin d'étude Master Academique , Université des sciences et de technologie, Mostaganem ,juin ,(2016)

6. Bouhamou.N., «Influence des paramètres de composition sur le comportement du béton autoplaçant vis -à vis du retrait » thèse de Doctorat, (2008)

7. José Oliveira.M, Shrinkage of self-compacting concrete. A comparative analysis CEPAC, University of the Algarve, Faro, Portugal, January (2017), Pages 117124

8. Mostefa. F, Étude du comportement d'un béton Autoplaçant à base de sédiment de dragage vis-à-vis du retrait, Mémoire de Magister, Université des sciences et de technologie, Mostaganem, 23Février, (2012).

9. Belaribi.O.,Comportement d'un béton autplaçant vis-vis des attaques chimiques, Thèse de Magister, Université des sciences et de la technologie d'Oran Mohamed Boudiaf U.S.T.O Oran,(2009) 